Canad. Math. Bull. Vol. 19 (2), 1976

\title{
COVER-AVOIDANCE PROPERTIES IN FINITE SOLUBLE GROUPS
}

\author{
BY \\ M. J. TOMKINSON
}

\begin{abstract}
We give a general method for constructing subgroups which either cover or avoid each chief factor of the finite soluble group $G$. A strongly pronormal subgroup $V$, a prefrattini subgroup $W$, an $\Im$-normalizer $D$ and intersections and products of $V, W$, and $D$ are all constructable. The constructable subgroups can be characterized by their cover-avoidance property and a permutability condition as in the results of J. D. Gillam [4] for prefrattini subgroups and $\mathfrak{F}$-normalizers.
\end{abstract}

There are three main types of subgroup which cover or avoid each chief factor in a finite soluble group. These are the $\mathfrak{\wp}$-normalizers, the prefrattini subgroups and the strongly pronormal subgroups. In this note we give a general method for constructing subgroups which either cover or avoid each chief factor so that each of the types of subgroup mentioned above is constructable.

G. A. Chambers [1] proved that a conjugacy class of strongly pronormal subgroups is characterized completely by the cover-avoidance property of the subgroups, i.e. by specifying which chief factors of $G$ are covered and which are avoided. J. D. Gillam [4] gave a permutability condition, which, together with the cover-avoidance property, characterized the $\mathfrak{\wp}$-normalizers and the prefrattini subgroups. We show that the constructable subgroups which we consider can be characterized as in Gillam's results. The permutability condition is automatically satisfied for strongly pronormal subgroups and so our result will imply the characterization of these three types of subgroup. The intersections and products of a strongly pronormal subgroup $\mathrm{V}$, a prefrattini subgroup $W$ and an $\mathfrak{f}$-normalizer $D$ are also constructable. These subgroups have been previously investigated in [2], [5] and [6].

In attempting to give a unified setting to the discussion of cover-avoidance properties, we introduced the concept of a SCAR-subgroup in [7]. We refer the reader to this paper for definitions and notation.

Our construction of $\mathbf{S} C A R$-subgroups will be based on subgroups of the form $N_{G}\left(Q \cap S_{p^{\prime}}\right)$, where $Q \triangleleft G$ and $S_{p^{\prime}}$ is a Sylow $p$-complement. It is well known (see e.g. [3] Lemma 3.1) that $N_{G}\left(Q \cap S_{p^{\prime}}\right)$ avoids the $Q$-eccentric $p$-chief factors of $G$ and covers all other chief factors.

Received by the editors June 13, 1975.

A.M.S. Classification: 20D10. 
LEMMA 1. Let $L$ and $Q$ be normal subgroups of the finite soluble group $G$ and let $H$ be a subgroup normalized by $S_{p^{\prime}}$. Then

$$
H \cap L N_{G}\left(Q \cap S_{p^{\prime}}\right)=(H \cap L) N_{H}\left(Q \cap S_{p^{\prime}}\right) \text {. }
$$

Proof. It is clear that $H \cap L N_{G}\left(Q \cap S_{p^{\prime}}\right) \geqslant(H \cap L) N_{H}\left(Q \cap S_{p^{\prime}}\right)$. Conversely, using [3] Lemma 2.12, we have

$$
\begin{aligned}
H \cap L N_{G}\left(Q \cap S_{p^{\prime}}\right) & =N_{H}\left(L Q \cap L S_{p^{\prime}}\right) \\
& \leqslant N_{H}\left((H \cap L) Q \cap H S_{p^{\prime}} \cap L S_{p^{\prime}}\right)
\end{aligned}
$$

As in [8] Lemma 4.8, for example, $H S_{p^{\prime}} \cap L S_{p^{\prime}}=(H \cap L) S_{p^{\prime}}$ and so

$$
H \cap L N_{G}\left(Q \cap S_{p^{\prime}}\right) \leqslant N_{H}\left((H \cap L) Q \cap(H \cap L) S_{p^{\prime}}\right)=(H \cap L) N_{H}\left(Q \cap S_{p^{\prime}}\right) .
$$

It should be noted that the condition $S_{p^{\prime}} \leqslant N_{G}(H)$ is necessary in the above Lemma. For if $G$ is the symmetric group of degree 3, $Q=G, L=G^{\prime}$ and $H$ and $S_{p^{\prime}}$ are distinct subgroups of order 2 , then $H \cap L N_{G}\left(S_{p^{\prime}}\right)=H$, whereas $(H \cap L) N_{H}\left(S_{p^{\prime}}\right)=1$.

THEOREM 2. Let $L_{i}, Q_{i}(i=1, \ldots, n)$ be normal subgroups of the finite soluble group G. Then $\bigcap_{i=1}^{n} L_{i} N_{G}\left(Q_{i} \cap S_{p^{\prime}}\right)$ is a CAR-subgroup of $G$. A p-chief factor $U / V$ is avoided if there exists $i_{1}, \ldots, i_{k}$ such that $\bigcap_{j=1}^{k} L_{i_{j}}$ avoids $U / V$ and $U / V$ is $Q_{i_{j}}$-eccentric for each $j=1, \ldots, k$; otherwise $U / V$ is covered.

Proof. Let $U / V$ be a $p$-chief factor of $G$. By Lemma 1, $U \cap$ $\bigcap_{i=1}^{n} L_{i} N_{G}\left(Q_{i} \cap S_{p^{\prime}}\right)=\bigcap_{i=1}^{n}\left(U \cap L_{i}\right) N_{U}\left(Q_{i} \cap S_{p^{\prime}}\right)$. If $\left(U \cap L_{i}\right) N_{G}\left(Q_{i} \cap S_{p^{\prime}}\right) \leqslant V$, for any $i$, then $U / V$ is clearly avoided. So we may assume that for each $i$, either $\left(U \cap L_{i}\right) V=U$ or $U / V$ is $Q_{i}$-central ([7], Corollary 2.4). Renumbering if necessary, we may suppose that $U / V$ is $Q_{i}$-eccentric for $i=1, \ldots, r$, and $Q_{i}$-central for $i=r+1, \ldots, n$. If $\bigcap_{i=1}^{r}\left(U \cap L_{i}\right)$ covers $U / V$, then $\bigcap_{i=1}^{n}(U \cap$ $\left.L_{i}\right) N_{U}\left(Q_{i} \cap S_{p^{\prime}}\right) \geqslant \bigcap_{i=1}^{r}\left(U \cap L_{i}\right) \cap N_{U}\left(Q_{r+1} \cdots Q_{n} \cap S_{p^{\prime}}\right)$ and this covers $U / V$ ([7], Corollary 2.4).

This leaves the situation in which $\bigcap_{i=1}^{r}\left(U \cap L_{i}\right) \leqslant V$. We prove by induction on $r$ that $\bigcap_{i=1}^{r}\left(U \cap L_{i}\right) N_{U}\left(Q_{i} \cap S_{p^{\prime}}\right) \leqslant V$. This is certainly true if $r=1$ so we may assume that $r>1$. Since $L_{1}$ covers $U / V$, we have $\left(U \cap L_{1}\right) /\left(V \cap L_{1}\right) \cong U / V$ and so $\left(U \cap L_{1}\right) /\left(V \cap L_{1}\right)$ is $Q_{i}$-eccentric for $i=2, \ldots, r$. Also $\bigcap_{i=2}^{r}\left(U \cap L_{1} \cap L_{i}\right) \leqslant$ $V \cap L_{1}$ and so, by induction, $L_{1} \cap \bigcap_{i=2}^{r}\left(U \cap L_{i}\right) N_{U}\left(Q_{i} \cap S_{p^{\prime}}\right) \leqslant L_{1} \cap V$. Writing $X$ for $\bigcap_{i=2}^{r}\left(U \cap L_{i}\right) N_{U}\left(Q_{i} \cap S_{p^{\prime}}\right)$, we have $L_{1} \cap X \leqslant V$ and, by Lemma 1 , $\bigcap_{i=1}^{r}\left(U \cap L_{i}\right) N_{U}\left(Q_{i} \cap S_{p^{\prime}}\right)=\left(U \cap L_{1}\right) N_{U}\left(Q_{1} \cap S_{p^{\prime}}\right) \cap X=\left(L_{1} \cap X\right) N_{X}\left(Q_{1} \cap S_{p^{\prime}}\right)$ $\leqslant V$, as required.

Theorem 2.3 of [7] immediately gives the following

Corollary 3. If $M, N, L_{i}, Q_{i}(i=1, \ldots, n)$ are normal subgroups of the finite soluble group $G$, then

$$
N\left\{M S_{p^{\prime}} \cap \bigcap_{i=1}^{n} L_{i} N_{G}\left(Q_{i} \cap S_{p^{\prime}}\right)\right\}
$$

is a CAR-subgroup of $G$ containing $S_{p^{\prime}}$. 
Now let $\mathbf{S}=\left\{S_{p^{\prime}}\right\}$ be a Sylow system of $G$ and, for each prime $p$, let $M^{(p)}$, $N^{(p)}, L_{i}^{(p)}, Q_{i}^{(p)}(i=1, \ldots, n(p))$ be normal subgroups of $G$. If

$$
B_{p}=N^{(p)}\left\{M^{(p)} S_{p^{\prime}} \cap \bigcap_{i=1}^{n(p)} L_{i}^{(p)} N_{G}\left(Q_{i}^{(p)} \cap S_{p^{\prime}}\right)\right\}
$$

then $\mathscr{B}=\left\{B_{p}\right\}$ is a $\mathbf{S} C A R$-system and $B=\bigcap_{p} B_{p}$ is a $\mathbf{S C A R}$-subgroup. We shall call a $\mathbf{S} C A R$-subgroup of this type constructable. Note that each $B_{p}$ is itself a constructable $\mathbf{S} C A R$-subgroup.

Lemma 4. Let $B$ be a constructable $\mathbf{S C A R}$-subgroup of $G$ containing $S_{p^{\prime}}$. Let $X$ be a subgroup containing $S_{p^{\prime}}$ which avoids each $B$-avoided chief factor of $G$. Then $X \leqslant B$.

Proof. By induction on $|G|$. Let $B=N\left\{M S_{p^{\prime}} \cap \bigcap_{i=1}^{n} L_{i} N_{G}\left(Q_{i} \cap S_{p^{\prime}}\right)\right\}$. $X N / N$ avoids each chief factor of $G / N$ avoided by the constructable SCAR-subgroup $B / N$. If $N \neq 1$, then, by induction, $X \leqslant X N \leqslant B$.

So we may assume that $N=1$. Since $X$ avoids each $p$-chief factor above $M$, we have $X \leqslant M S_{p^{\prime}}$. It remains to show that $X \leqslant L_{i} N_{G}\left(Q_{i} \cap S_{p^{\prime}}\right)$. If $L_{i} \neq 1$ then $X L_{i} / L_{i}$ avoids the $Q_{i}$-eccentric chief factors of $G / L_{i}$ and so, by induction, $X \leqslant X L_{i} \leqslant L_{i} N_{G}\left(Q_{i} \cap S_{p^{\prime}}\right)$. So we may assume that $L_{i}=1$.

Let $A$ be a minimal normal subgroup of $G$ so that, by induction, $X \leqslant X A \leqslant$ $A N_{G}\left(Q_{i} \cap S_{p^{\prime}}\right)$. If $A \leqslant N_{G}\left(Q_{i} \cap S_{p^{\prime}}\right)$ then $X \leqslant N_{G}\left(Q_{i} \cap S_{p^{\prime}}\right)$ and so we may assume that $A \cap N_{G}\left(Q_{i} \cap S_{p^{\prime}}\right)=1$. Therefore $X=X \cap N_{G}\left(A Q_{i} \cap A S_{p^{\prime}}\right) \leqslant$ $N_{X}\left(A Q_{i} \cap X \cap A S_{p^{\prime}}\right)=N_{X}\left(A Q_{i} \cap S_{p^{\prime}}\right) \leqslant N_{G}\left(Q_{i} \cap S_{p^{\prime}}\right)$, as required.

THeOREM 5. Let $B=\bigcap_{p} B_{p}$ be a constructable SCAR-subgroup. A subgroup $X$ of $G$ is conjugate to $B$ if and only if $X$ satisfies the conditions:

(i) $X$ covers and avoids the same chief factors as $B$,

(ii) there is a Sylow system $\mathbf{T}=\left\{T_{p^{\prime}}\right\}$ of $G$ such that $X T_{p^{\prime}}$ is a subgroup of $G$ for all $T_{p^{\prime}} \in \mathbf{T}$.

Proof. First suppose that $X=B^{g}$ and let $\mathbf{T}=\mathbf{S}^{g}$. Then $X T_{p^{\prime}}=\left(B S_{p^{\prime}}\right)^{g}=B_{p}^{g}$ is a subgroup.

Conversely, suppose $X T_{p^{\prime}}$ is a subgroup for all $T_{p^{\prime}} \in \mathbf{T}=\mathbf{S}^{\text {g. Then }} X T_{p^{\prime}}$ has the same cover-avoidance properties as $B_{p}$. By Lemma $4, X T_{p^{\prime}} \leqslant B_{p}^{\mathrm{g}}$. Thus $X \leqslant$ $\bigcap_{p} B_{p}^{g}=B^{g}$ and order considerations give $X=B^{\mathrm{g}}$.

It is clear that $\mathfrak{\wp}$-normalizers are constructable $C A R$-subgroups. So also are $\mathscr{X}$-prefrattini subgroups since an abnormal $p$-maximal subgroup $A$ has the form $L N_{G}\left(Q \cap S_{p^{\prime}}\right)$, where $L=\operatorname{core}_{G} A$ and, if $C / L=$ Fitt $(G / L)$, we may take $Q / C$ to be $O_{p^{\prime}}(G / C)$. (e.g. [7] Lemma 3.1). We may take $M^{(p)}$ to be the intersection of the normal $p$-maximal subgroups. 
Strongly pronormal subgroups are obtained by putting $B_{p}=M^{(p)} S_{p^{\prime}}$ for each p.

It can also be seen easily, from [7] for example, that all intersections and products of $V, W$ and $D$ are constructable SCAR-subgroups.

We have not used induction on $|G|$ until Lemma 4 and so the earlier results can also be proved in the class $\mathfrak{U}$ (see [3]) although it is not clear whether we could take an infinite intersection $\bigcap_{i \in I} L_{i} N_{G}\left(Q_{i} \cap S_{p^{\prime}}\right)$ in Theorem 2. Without this, of course, we would not include the prefrattini subgroups.

Even if constructable SCAR-subgroups could be satisfactorily defined in $\mathfrak{U}$ it would still not be possible to extend the characterization given in Theorem 5 . The discussion of an example of B. Hartley given in Section 4 of [7] shows that this cannot be done.

\title{
REFERENCES
}

1. G. A. Chambers, p-Normally Embedded Subgroups of Finite Soluble Groups, J. Alg. 16 (1970) 442-455.

2. G. A. Chambers, On f-prefrattini subgroups, Canadian Math. Bull. 15 (1972) 345-348.

3. A. D. Gardiner, B. Hartley, and M. J. Tomkinson, Saturated Formations and Sylow Structure in Locally Finite Groups, J. Alg. 17 (1971) 177-211.

4. J. D. Gillam, Cover-avoid subgroups in finite solvable groups, J. Alg. 29 (1974) 324-329.

5. T. O. Hawkes, Analogues of prefrattini subgroups, Proc. Internat. Conf. Theory of Groups. Austral. Nat. Univ. Canberra, August 1965, pp. 145-150 (Gordon and Breach, New York 1967).

6. A. R. Makan, On certain sublattices of the lattice of subgroups generated by the prefrattini subgroups, the injectors and the formation subgroups, Canad. J. Math. 25 (1973) 862-869.

7. M. J. Tomkinson, Prefrattini subgroups and cover-avoidance properties in $\mathfrak{U}$-groups, Canad. J. Math. 27 (1975) 837-851.

8. M. J. Tomkinson, Formations of locally soluble FC-groups, Proc. London Math. Soc. (3) 19 (1969) 675-708.

\author{
Department of Mathematics \\ UNIVERSITY OF GLASGOW \\ GLASGOW G12 8QW
}

